\title{
SPATIO-TEMPORAL DYNAMICS OF CHAIN OF COUPLED IMPACT PENDULUMS
}

\author{
R. STĘPIEŃ AND R.A. KosińsKi \\ Institute of Physics, Warsaw University of Technology \\ Koszykowa 75, 00-662 Warsaw, Poland
}

(Received February 12, 1997; in final form April 25, 1997)

A chain of $N$, one-dimensional, impact pendulums is investigated using numerical solutions of equations of motion. Ranges of periodic, mixed and chaotic motion are found using spatio-temporal diagrams. A correlation function and power spectrum density functions are calculated for these digitized diagrams. Influence of the coupling strength $k$ and the number of pendulums $N$ on the dynamics of the system is discussed.

PACS numbers: $05.45 .+b$

\section{Introduction}

Simple models of a spatially extended system consisting of a set of coupled oscillators or pendulums allow us to investigate many phenomena, starting from solid state physics up to population biology [1-4], occurring in the real physical systems. In particular, in the set of the coupled limit cycle oscillators with different natural frequencies distributed across the system [5], it is possible to observe a nonlinear time evolution, in which the collective synchronization appears $[1,2]$. Another application of the set of coupled limit cycle oscillators is connected with an investigation of chemical oscillating reactions $[3,6,7]$. A system of chaotic oscillators was used in Ref. [8] for the observation of localised structures like solitons and domain walls, which may create far off equilibrium in a nonlinear medium. Abraham [9] analysed the dynamics of a linear chain of damped-driven chaotic pendulums, in which very rich and complex dynamics of the system, both in space and time, was found.

In the present paper the dynamics of a chain of coupled, 1D pendulums with the limited range of its motion caused by the hard walls located between each two pendulums, is investigated. Such a type of pendulum is called an impact oscillator and was analysed earlier by Isömaki and von Böhm [10,11]. It was found that the dynamics of a single impact oscillator has a rather complex character [10-12], thus the dynamics of a spatially extended system consisting of $N$ linear coupled impact pendulums seems to be an interesting problem and will be analysed in the present work. 


\section{Model}

The chain of one-dimensional, coupled impact pendulums was considered (see Fig. 1). The equations of motion for such a system are as follows:

$$
\begin{aligned}
& \ddot{\Theta}_{j}+\alpha \dot{\Theta}_{j}+\omega^{2} \sin \left(\Theta_{j}\right)+k\left(2 \Theta_{j}-\Theta_{j+1}-\Theta_{j-1}\right)=A \cos (\Omega t), \\
& j=1, \ldots, N-1
\end{aligned}
$$

with the conditions for the impacts of pendulums with two hard walls, located between each two pendulums in $\Theta=0$ and $\Theta=\pi$, which cause the change of a sign of pendulum's velocity

$$
\left\{\begin{array}{c}
\Theta_{j}\left(t_{i}^{-}\right)=0 \rightarrow \dot{\Theta}_{j}\left(t_{i}^{+}\right)=-\dot{\Theta}_{j}\left(t_{i}^{-}\right), \\
\text {or } \\
\Theta_{j}\left(t_{i}^{-}\right)=\pi \rightarrow \dot{\Theta}_{j}\left(t_{i}^{+}\right)=-\dot{\Theta}_{j}\left(t_{i}^{-}\right) .
\end{array}\right.
$$

Here, $j=1, \ldots, N$, is a number of a pendulum, $\Theta_{j}$ - the angular displacement of $j$-th pendulum, $A-$ the amplitude of the external oscillatory force, $\Omega-$ the frequency of the external force, $\alpha$ - the damping parameter, $\omega$ - the natural frequency of a single pendulum, $k$ - the coupling strength between two nearest neighbours of the $j$-th pendulum. A dot over a symbol denotes the time derivative. The values of the parameters of pendulums, chosen for our computations were: $A=7.7, \Omega=\pi, \alpha=0.25, \omega=4.21$. The periodic boundary conditions and random initial condition for $\Theta_{j}$ (restricted to $0 \leq \Theta_{j} \leq \pi$ ) and $\dot{\Theta}_{j}$ were assumed.

The system of equations $(1,2)$ was solved numerically using a predictor-corrector as algorithm [13]. The time points of impacts were found from the boundary conditions (2), using cubic spline interpolations with a high accuracy [14]. The computations were rather time consuming and were performed with a CRAY CS 6400 superserver.

For the presentation of dynamics of the considered system, spatio-temporal diagrams were constructed $[15,16]$. The time evolution of each $\Theta_{j}(t), j=1, \ldots, N$, obtained from the numerical solutions of equations of motion $(1,2)$, was digitized using the following rule. In each $n$-th time step of the integration procedure the values $(-1)$ and $(+1)$ were assigned to the $i$-th grid point, if $\left|\Theta_{j}\right|<|\bar{\Theta}|$ or $\left|\Theta_{j}\right| \geq|\bar{\Theta}|$, respectively. Here $\bar{\Theta}$ denotes the spatial a verage of $\Theta$ over all pendulums in the chain. Thus, the information contained in the solutions of Eqs. $(1,2)$ was reduced, however, a type of the motion of the pendulums was maintained in the time series of $(-1)$ and $(+1)$ of each pendulum which appeared instead of the sequence of solutions $\Theta_{j}(\Delta t=n \Delta T)$. The spatio-temporal diagrams for the motion of the chain of pendulums were constructed as a sequence of white (for -1$)$ and black (for +1 ) cells (see Fig. 2) [17].

For the investigation of the dynamical properties of the system a harmonic analysis of time evolution of a spatial average $\bar{\Theta}(t)$ was used. It was based on the calculation of power spectrum density (PSD) estimated by the periodogram $[18,17]$. Additionally, for the detail information about the dynamics of the system the correlation function between two chosen ( $l$-th and $m$-th) pendulums in the chain was calculated

$$
C(\tau)=\sum_{k=0}^{L-1} a_{k} b_{k+\tau},
$$



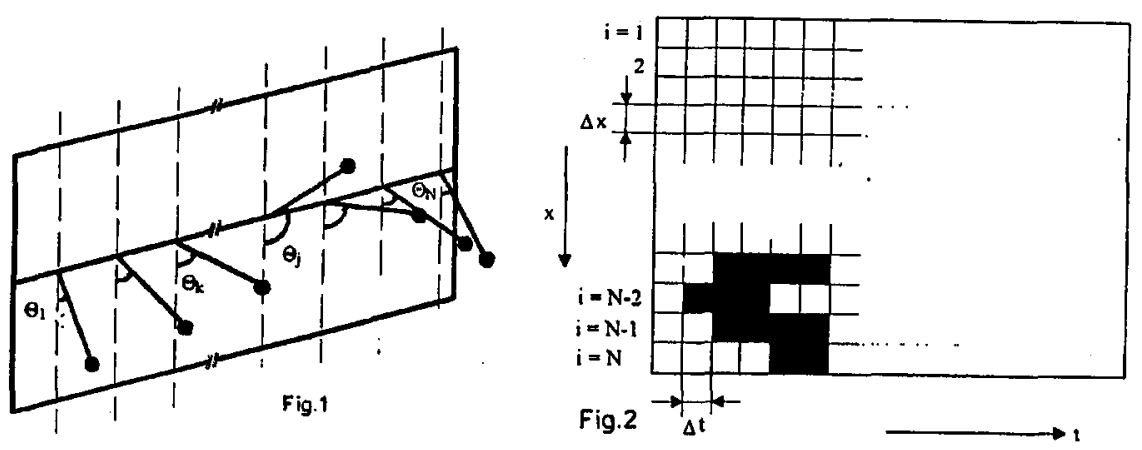

Fig. 1. Model of a chain of $N$ impact pendulums; $\Theta_{j}$ is the angular displacement of $j$-th pendulum.

Fig. 2. Construction of spatio-temporal diagram. The dimensions of one numerical cell are $(\Delta x, \Delta t)$; black and white cell are denoted as +1 and -1 , respectively.

where $a_{k}$ is the sequence of "- 1 " and " +1 " coming from the part of the spatio-temporal diagram referring to the pendulum $j=l, b_{k}$ is the sequence of " -1 " and " +1 " connected with the pendulum $j=m$, the amount of samples is denoted $L$ and $\tau$ is the time shift. The correlation between time series coming from two pendulums chosen in the chain, enable us to find information about synchronization in the system.

\section{Results and discussion}

The equations of motion $(1,2)$ were solved numerically for different values of control parameters: the coupling strength $k$ and the number of pendulums in the chain $N$. Three types of motion were found: periodic, mixed and chaotic.

\section{TABLE}

Ranges of the types of motion of the system.

\begin{tabular}{c|c}
\hline \hline Limits of $k$ & Type of motion \\
\hline $0 \leq k<0.031$ & Periodic \\
$0.031<k \leq 0.051$ & Mixed \\
$k>0.051$ & Chaotic
\end{tabular}

Let us consider the influence of the coupling strength $k$ on the dynamics of the system. For $N=64$ the ranges of these three types of motion are shown in Table. As we see the periodic motion is observed for the smallest values of the coupling $k$. A spatio-temporal diagram for this range is shown in Fig. 3. All pendulums oscillate with the same frequency, but they have different phases, which results from the random initial conditions. The periodic character of the motion is easily seen as a regular pattern on the diagram. Confirmation of the periodic character of the motion gives the calculation of the correlation function $C$, shown 

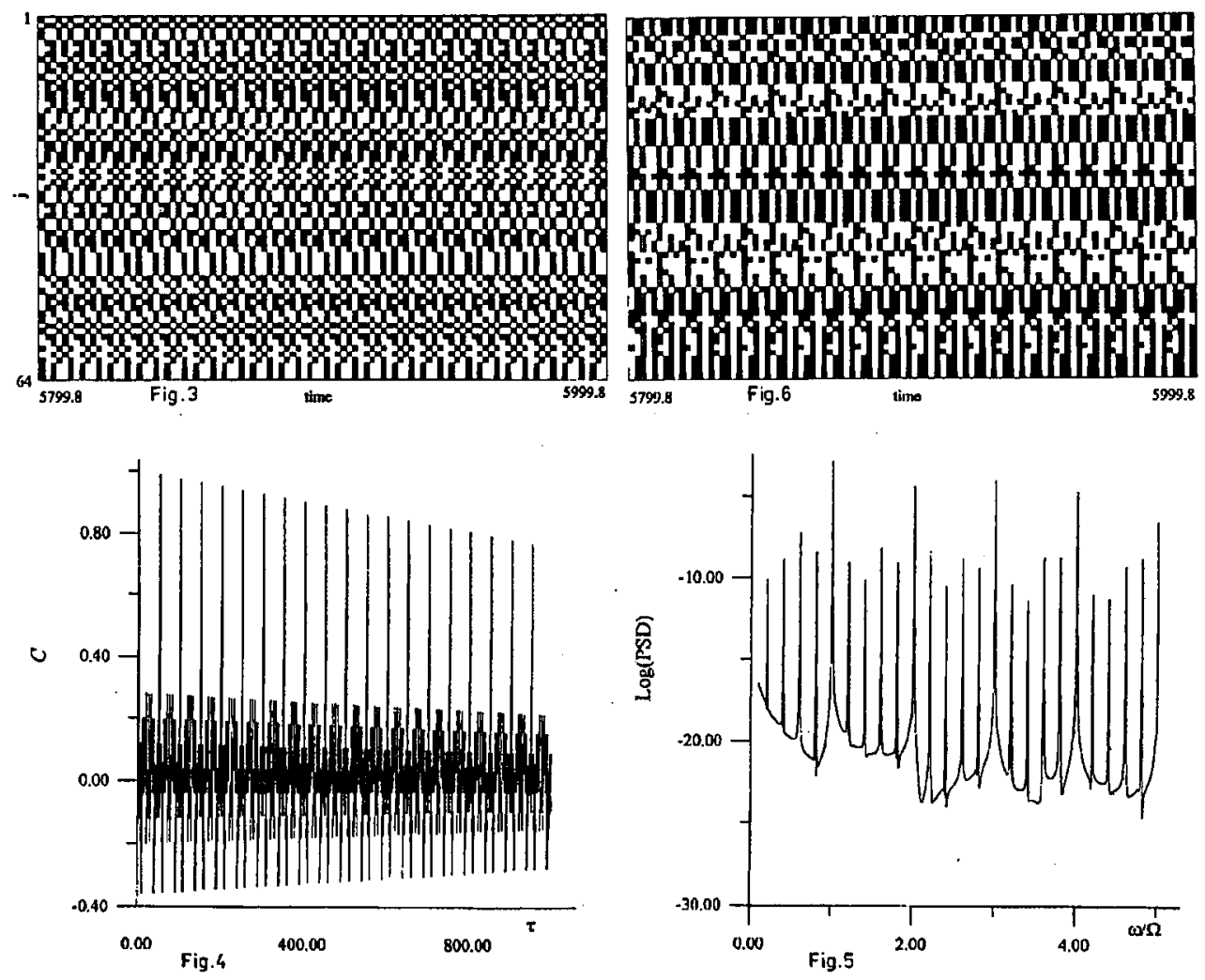

Fig. 3. Spatio-temporal diagram for the periodic type of motion of the system with $N=64$ and $k=0$.

Fig. 4. The correlation function $C$ as a function of a time shift $r$ between pendulums $l=1$ and $m=32$ for $N=64$ and $k=0$.

Fig. 5. Logarithm of the relative power spectrum density function (PSD) as a function of relative frequency $\omega / \Omega$ for the system in the periodic motion, with $N=64$ and $k=0$ ( $\Omega$ is the external force frequency).

Fig. 6. Spatio-temporal diagram for the mixed type of motion for the system with $N=64$ and $k=0.05$.

in Fig. 4. The maxima of $C$ are observed for every 5 periods of external force $\Omega / 2 \pi$. Additionally, the results of calculation of PSD for the locations of all pendulums averaged over $N$, are shown in Fig. 5. On the horizontal axis is the reduced frequency ( $\Omega$ is the frequency of the external force) and on the vertical axis is the logarithm of PSD. The highest peak refers to the frequency of the external force $\Omega$, the first peak corresponds to the frequency $\Omega / 5$, while the other peaks correspond to the harmonics of this frequency. Such a location of peaks is a result of a specific distance between hard walls, defined in Eq. (2).

The mixed type of motion is observed for $k>0.031$ (see Table). In this type of motion a part of pendulums oscillates with the frequency other than that in the 
pcriodic motion, this is seen on a spatio-temporal diagram shown in Fig. 6. The diagram has also a rather regular character, however some pendulums have the period other than $\Omega / 2 \pi$. At the correlation function for this type of motion (see Fig. 7) additional peaks, in comparison to the correlation function for the periodic motion (cf. Fig. 4), appear. They are due to the additional frequency of the part of oscillators. Also a number of rather small peaks connected with these additional frequencies occurs at PSD (see Fig. 8). It is interesting that the route to chaos for the present system occurs via the mixed type of motion, which differs from the routes to chaos known in the literature (via the period doubling, quasiperiodic motion or intermittence) [19]. A similar route to chaotic motion was found earlier by Abraham [9] for the system of chain of coupled dumped chaotic pendula.
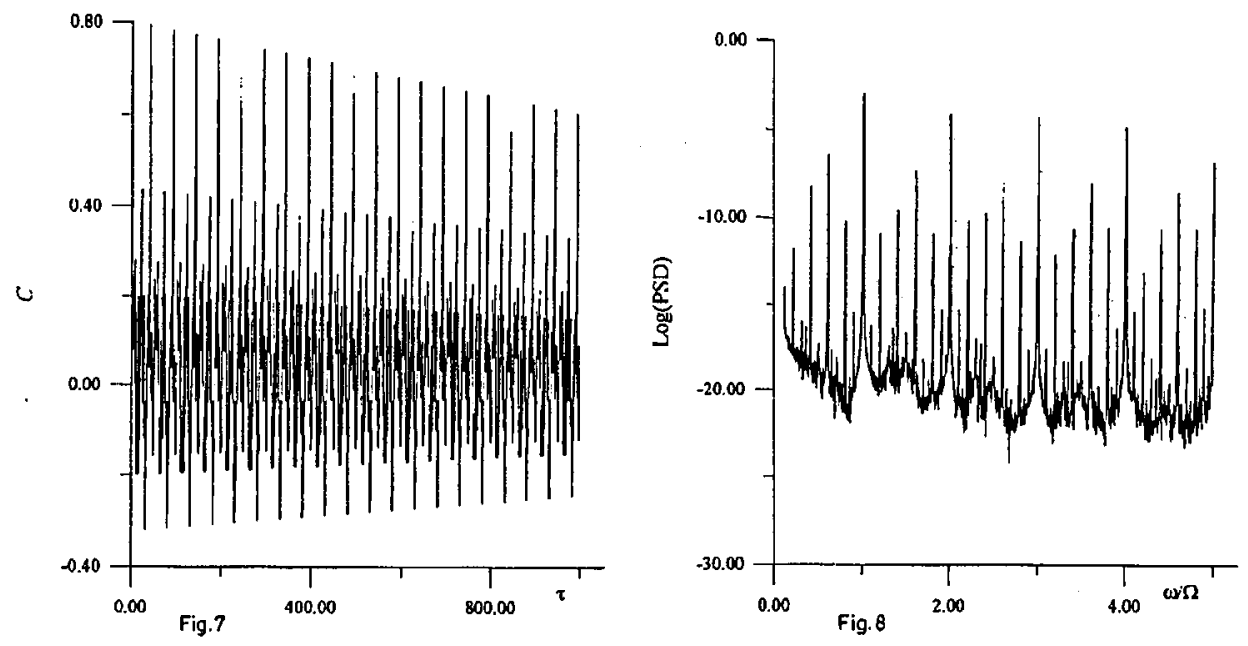

Fig. 7. The correlation function $C$ as a function of a time shift $\tau$ between pendulums $l=1, m=31$ for the system in the mixed type of motion, with $N=64$ and $k=0.05$.

Fig. 8. Logarithm of the relative power spectrum density function (PSD) as a function of relative frequency $\omega / \Omega$ for the system in the mixed type of motion with $N=64$ and $k=0.05$.

The chaotic motion of the system appears for $k>0.051$. In this range irregular oscillations of all pendulums are observed, which is seen on a spatio-temporal diagram (Fig. 9). One can find, however, time windows in which some pendulums oscillate regularly. The correlation function and PSD for this range of motion are shown in Fig. 10 and 11, respectively, and they have a character confirming the chaotic motion of the system. The high peaks connected with the external force frequency and its harmonics are still visible.

Calculations of the dynamics of the system were also performed for a significantly shorter chain of pendulums with $N=4$. Also in this case three types of motion were found. It is interesting, however, that the limits of the periodic, mixed and chaotic motion are approximately the same as for the case of the long 


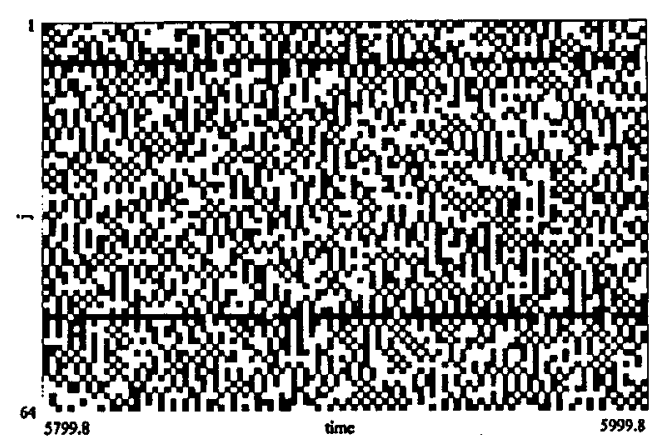

Fig. 9. Spatio-temporal diagram for the chaotic type of motion for the system with $N=64$ and $k=0.1$.
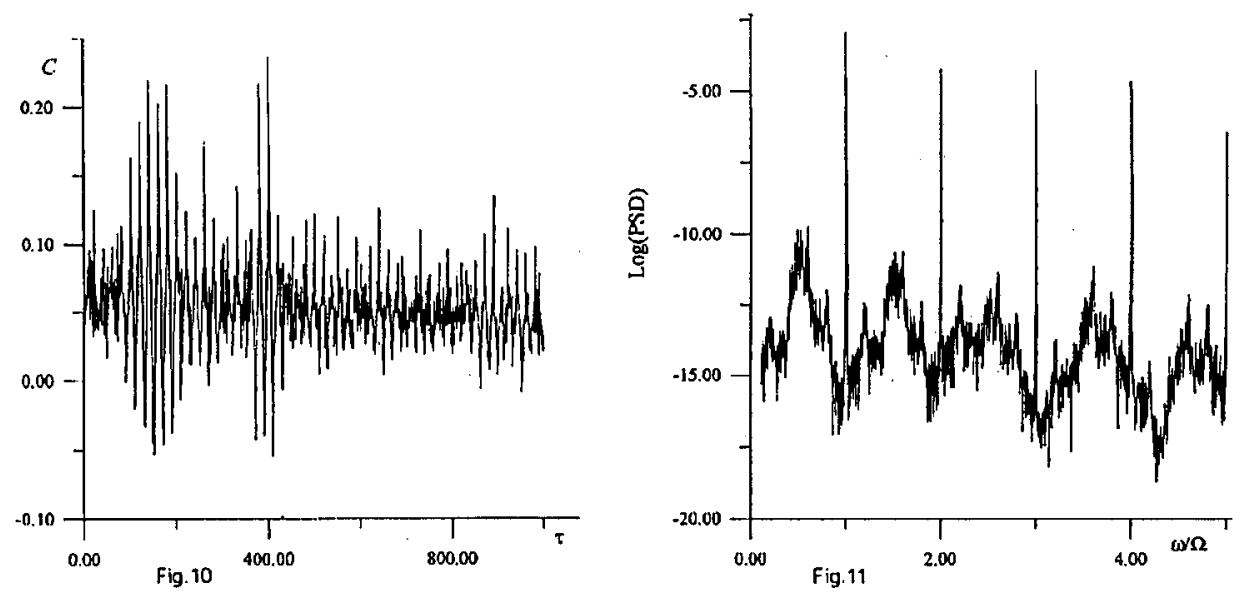

Fig. 10. The correlation function $C$ as a function of a time shift $\tau$ between pendulums $l=1$ and $m=32$ for the system in the chaotic motion, with $N=64$ and $k=0.1$.

Fig. 11. Logarithm of the relative power spectrum density function (PSD) as a function of relative frequency $\omega / \Omega$ for the system with $N=64$ and $k=0.1$.

chain with $N=64$ pendulums. The differences of the critical values of $k$ between the case with $N=4$ and the case with $N=64$ are smaller than 0.001 .

It was found that a chain of coupled impact pendulums have interesting dynamic properties. Pendulums may oscillate regularly, or, if the coupling between them is large enough, the motion of the system is chaotic. In this system the route from a periodic to a chaotic motion is realised via a mixed type of motion, in which some pendulums oscillate with new frequencies, other than the frequency of the majority of pendulums. When the value of the coupling $k$ increases the number of pendulums with such frequencies increases also, which finally results in a chaotic motion of the system.

A small influence of the size $N$ of the chain on its dynamics suggests that the influence of the impacts of pendulums with the hard walls is significantly larger 
than the influence of the coupling between the pendulums. It is interesting in our system that even in the case of rather high values of the coupling between the pendulums the synchronization of their motion does not occur.

The hard walls in our model have a constant localization (in $\Theta=0$ and $\Theta=\pi$ ), which determines the motion of each pendulum very significantly. Another localization of hard walls (e.g. $\Theta=\pi / 2$ and $\Theta=3 \pi / 2$ ) would have a smaller influence on the motion of pendulums, especially for a small amplitude of an external force $A$. In such a case, the influence of the coupling between the pendulums on its dynamics would be more important than in the present case.

\section{References}

[1] A.T. Winfree, The Geometry of Biological Time, Springer Verlag, Berlin 1980.

[2] A.T. Winfree, J. Theor. Biol. 16, 15 (1967).

[3] Y. Kuramoto, Chemical Oscillations, Waves and Turbulence, Springer Verlag, Berlin 1984.

[4] Formation Dynamics and Slatistics of Palterns, Eds. K. Kawasaki, M. Suzuki, A. Onuki, World Science, Singapore 1990.

[5] P.C. Matthews, S. Strogatz, Phys. Rev. Lett. 65, 1701 (1990).

[6] II. Daido, Phys. Rev. Lett. 61, 231 (1988).

[7] H. Daido, Phys. Rev. Lett. 68, 1073 (1992).

[8] A. Greenfield, S. Putterman, W. Wright, Phys. Lett. A 185, 321 (1993).

[9] F.A. Abraham, Phys. Rev. E 47, 1625 (1993).

[10] II.M. Isömaki, J. von Böhm, R. Räty, Phys. Lett. A 126, 484 (1988).

[11] H.M. Isömaki, J. von Böhm, R. Räty, Phys. Lett. A 107, 343 (1985).

[12] E.A. Jackson, Perspectives of Nonlinear Dynamics: I and II, Cambridge University Press, Cambridge 1991.

[13] G.D. Byrne, A.C. Hindmarsh, ACM Trans. Malh. Software 1, 71 (1975).

[14] W.H. Press, B.P. Flannery, S.A. Teukolsky, W.T. Vetterling, Numerical Recipes, Cambridge Univ. Press, Cambridge 1986.

[15] K. Kaneko, Physica D 37, 60 (1989).

[16] R.A. Kosiński, Phys. Lett. A 169, 263 (1992).

[17] R.A. Kosiński, R. Stępień, Acta Phys. Pol. A 87, 575 (1995).

[18] F.J. Hariss, Proc. IEEE 66, 51 (1978).

[19] Hao Bai-Lin, Chaos, World Scientific Publ., Singapore 1984. 